\title{
Grundgeschichte und Chronik - Kontinuität und Diskontinuität in Altisraels Geschichtsschreibung
}

\section{Das Neue im Alten - die Fragestellung der biblischen Chronikbücher}

Die Geschichtsdarstellung von Chr wird durch die Menschheits- und Bürgerlisten (1 Chron 1:1-9:9 bzw. 10:14) zu Beginn und durch die abschliessende Feststellung „bis zur Königsherrschaft des Königtums von Persien“ (2 Chron 36:20) eindeutig begrenzt. Von diesem weitgespannten Rahmen her würde man eine Darstellung erwarten, die entlang der grossen Linien die Themen und Ereignisse erzählt, die diesen zeitlichen Rahmen ausfüllen.

Seltsam berührt nun allerdings, dass ganze Partien und Abschnitte dieser Geschichte eigenartig behandelt oder, noch auffälliger, schlicht übergangen scheinen. Und zwar sind das durchaus bedeutende Epochen, die eine Kennerin der Sache zu dem Urteil bringen: „The intermediate history of Israel, told in Exodus through 1Samuel, is not found in Chronicles. ... As a result, some of the most important events in the history of Israel, such as the descent into Egypt, the exodus, the revelation at Mount Sinai, the conquest of the land of Canaan, the settlement, and the period of Joshua, the judges, and Saul are not described.“ Folgt daraus: „There is no break, not even any 'history', between Jacob and salvation."1

In seiner Neuerzählung aus dem Quell der alten Überlieferung behandelt der Chronist also nur die sogenannt staatliche Zeit Israels, geprägt und überragt durch die Gestalt Davids. Dabei sind es ganz andere Gesichtspunkte, die ihn leiten als jene, die die alte „Davidshausgeschichte“ ausmachen.

\footnotetext{
1 Sara Japhet, “Conquest and Settlement in Chronicles,” JBL 98 (1979): 205-18, wieder abgedr. in: Japhet, From the Rivers of Babylon to the Highlands of Judah (Winona Lake: Eisenbrauns, 2006), 38-52. Die Zitate hier pp. 39 und $50 \mathrm{f}$.; sehr pointiert p. 50: „The story of the exodus is not told in Chronicles and ... even the references to the exodus ... are often omitted in the parallel texts in Chronicles.“
}

Thomas Willi, University of Greifswald 
Seit fast zweihundert Jahren stand unter den Voraussetzungen der von Wilhelm M. L. De Wette und Julius Wellhausen inaugurierten historisch-kritischen Sicht der Geschichte und vor allem der Literaturgeschichte des alten Israel ${ }^{2}$ die Frage im Vordergrund, wie die Chronik die Vergangenheit umgebaut habe - sous entendu - dass sie das im Dienst und im Interesse ihrer Gegenwart, ihrer Partei und der sie leitenden Vor-urteile getan habe.

\section{Mosezeit und Davidszeit}

Für den Chronisten als Historiker ist die Fragestellung aber eine ganz andere: Was unterscheidet die von ihm dargestellte Davids- und Königszeit von der Mosezeit, vor allem - aber nicht ausschliesslich - hinsichtlich des Kultes? Wenn David nach der chronistischen Erzählung den ,altneuen“ Dienst in Jerusalem einrichtet, bestätigt und bekräftigt - was ist daran alt, was ist neu?

1) Der Kult der Exodus- und Wüstenzeit galt demselben Gott, dem David nach chronistischer Darstellung in hervorragender Weise dient. Getragen wurde der alte Gottesdienst, den priesterschriftlichen Überlieferungen zufolge, von dem Israel der zwölf Stämme. Ihre Einheit zeigt sich in der Sicht von Chronik in der Folge gefährdet. Sie löst sich schliesslich unter Saul (1 Chron 10) nahezu völlig auf, bis sie - und hier beginnt der Chronist seine Erzählung - von David neu hergestellt wird. Das bahnt sich an in 1. Chronik 11:1 - ויקבצו כל־ישראל אל־דויד.

2) Während zweitens in den pentateuchischen Zeiten Israels Gottesdienst keinen festen Platz hat und die Lade im Heiligtum des Zelts der Begegnung mobil ist, zeichnet sich die neue Ära dadurch aus, dass Israels Gott und sein Kult dank

\footnotetext{
2 Einer kritischen Rekonstruktion trat Chr hauptsächlich als irritierendes und störendes Hindernis in den Weg, so dass es etwa Wilhelm Martin Leberecht De Wette in seinen Beiträge zur Einleitung in das Alte Testament I: Kritischer Versuch über die Glaubwürdigkeit der Bücher der Chronik mit Hinsicht auf die Geschichte der Mosaischen Bücher und Gesetzgebung (Halle: Schimmelpfennig, 1806) vorrangig um „Wegräumung der Nachrichten der Chronik“ gehen musste. Besonders argwöhnisch wurden dabei die Inhalte beäugt, die dem Chronisten am Herzen lagen und die Israel aus seinem Gottesverhältnis heraus neu bestimmen sollten. Für eine empathische Würdigung der Darstellung des Kults in Chr war ein Jahrhundert kaum disponiert, das auf den Kulturkampf und die Frontstellung gegen den "Ultramontanismus" zulief, der schon in Formulierungen von De Wette anzuklingen scheint. So ist hier gleich eingangs von „einer unpriesterlichen Freiheit des Cultus“ in den „BB. Samuelis und der Könige“ die Rede, während „die Chronik den Zustand des Gottesdienstes von David an ganz Mosaisch-levitisch“ darstelle (4f.).
} 
David den verheissenen und gebotenen, aber nicht identifizierten ${ }^{3}$ festen „Ort“”4 gefunden haben: das Haus zu Jerusalem.

3) Sowohl in der Wüste wie nun in Jerusalem sind drittens Gottesdienst und Leben Israels durch die Anweisungen JHwHs geregelt. Der Jerusalemer Tempel ist freilich gewiss kein „Sanctuary of silence“,, 5 sondern ist vom Wort begleitet und erfüllt. Nachdem dieses am Sinai Mose mündlich gegeben wurde, wird sich das Geschehen von jetzt an ככתוב, ,wie geschrieben, schriftkonform“ vollziehen.

Während also [ad 1.] eine eindeutige Kontinuität zwischen Wüsten- und Davidszeit in dem ein-einzigen Gott besteht, dem der Dienst gilt, so leitet David nun anderseits [ad 2.] einen Übergang vom beweglichen Zelt zum festen Haus ein. Den Wendepunkt von der früheren Epoche zum neuen Zeitalter - der nicht etwa mit der Landnahme zusammenfällt - beschreibt das Kapitel 1. Chronik 17. ${ }^{6}$ Davids Plan, JHwH ein Haus zu bauen, erfährt in 1. Chronik nicht minder eine Absage als in 2. Samuel 7. Die Zusage, dass JHwH selbst es bauen werde, bekommt aber eine völlig andere Ausrichtung und Zielsetzung. Das „Haus“ ist nicht im übertragenen Sinne das (davididische) Königshaus wie im DtrG, sondern es handelt sich um ein reales Haus. ${ }^{7}$ Als „Gottes-Haus“ weist der künftige Tempel zu Jerusalem allerdings

3 Vgl. Stellen wie Ex 15:17; Dtn 12:5, 14.

4 Zur Bedeutung des מקום in Chr vgl. 1 Chron 13:11; 14:11, sodann in 1 Chron 15:1, 3, und zwar als Einleitung zu 1 Chron 16, hier vor allem V. 27, sodann über das diesbezüglich entscheidende Kapitel 1 Chron 21 hinaus bis hin zum Zielvers 2 Chron 3:1. Dazu Thomas Willi, "Die Suche nach dem Ort in der Chronik: Eine U-topie?,” in Worlds that could not be. Utopia in Chronicles, Ezra and Nehemiah, ed. Stephen J. Schweitzer and Frauke Uhlenbruch, LHBOTS 620 (London: Bloomsbury, 2016), 183-92.

5 Yehezkel Kaufmann, History of the religion of Israel, Bd. 2 (Jerusalem: Hebrew University, 1977), 477 f.; vgl. dazu Israel Knohl, The Sanctuary of Silence: The Priestly Torah and the Holiness School (Minneapolis: Fortress Press, 2005), 148-52.

6 Zur Doppelheit des - mosaischen - Zelts in Gibeon und dem Zelt, in dem David die Lade in Jerusalem unterbrachte, s. Gary N. Knoppers, "Hierodules, Priests, or Janitors? The Levites in Chronicles and the History of the Israelite Priesthood," JBL 118 (1999): 61; Jaeyoung Jeon, “The Priestly Tent of Meeting in Chronicles: Pro-Priestly or Anti-Priestly,” JHS 18, Art. 7 (2018): 1-15. Nach dem oben Ausgeführten handelt es sich aus chr Perspektive weniger um ein Entweder Oder denn um eine Übergangssituation, in der Salomo das von Mose überkommene „Zelt“ nach 2 Chron 1:3f. zu Gibeon aufsucht, während die Lade gleichzeitig in dem ihr von David behelfsmässig errichteten „Zelt“ zu Jerusalem aufbewahrt ist. Die Situation ergibt sich dem Chr durch die ihm vorliegenden Angaben in der Tradition.

7 Dazu ausführlich Thomas Willi, Gibt es in der Chronik eine "Dynastie Davids”? Ein Beitrag zu Semantik von bajit: FS E. Jenni, AOAT 336, ed. Jürg Luchsinger, Hans-Peter Mathys und Markus Saur (Münster: Ugarit-Verlag, 2007), 393-403 = ders., Israel und die Völker. Studien zur Literatur und Geschichte Israels in der Perserzeit, SBS 55, ed. Michael Pietsch (Stuttgart, Katholisches Bibelwerk, 2012)183-95, und vor allem ders., Chronik, Biblischer Kommentar (BK) XXIV/2.3 (Göttingen: Neukirchener Verlag in de Gruyter, 2019) 164-67, 181, 187-89. 
übermenschliche, ja überirdische Dimensionen auf, denn der Bau-Herr ist kein anderer als der König und Herr der Schöpfung selber, der von hier aus sein Weltkönigtum über Israel und über die Völkerwelt ausübt. ${ }^{8}$

\section{Mose in Davids Israel}

Bei allen damit eintretenden Veränderungen ist der Kult am künftigen Tempel $\mathrm{zu}$ Jerusalem keine komplette und radikale Neuerung, sondern er geschieht bei allen Unterschieden nach den Standards der alten - als solcher in Chronik nicht thematisierten - Früh- oder Ursprungszeit, chronistisch gesprochen ,gemäss der Tora Moses“. Mose wird in Chronik 21mal erwähnt, und zwar in unterschiedlicher, aber insgesamt doch zusammenlaufender Perspektive. An erster Stelle ist dabei der genealogische Gesichtspunkt zu nennen: 1. Chronik 5:29 und 23:13 wird er dezidiert als Sohn Amrams angeführt, diesbezüglich auf einer Ebene mit seinem älteren Bruder Aaron und seiner jüngeren Schwester Mirjam. Bereits darin verrät sich der genealogisch interessierte historische Zugang des Chronisten zur Mosegestalt. Aussagekräftig ist sodann die Näherbestimmung durch den עבד-Titel in 1. Chronik 6:34; 2. Chronik 1:3; 24:6, 9. Die drei Vorkommnisse sind anders gelagert als das Gros der 36 Belege in den mehrheitlich (nach-)deuteronomistischen Stellen des MT, wo er meist den "Gesetzesmittler" bezeichnet. ${ }^{9}$ Auf Mose als „Gottesknecht“ gehen in Chronik die alten Opfer- und weitergehenden Anordnungen zurück (2 Chron 1:3; 24:6, 9), ja er steht „mit Hilfe der Nennung עבד עיש איש אלהים ditels“ explizit für „die Wüstentradition““. 1. Chronik 23:14 f. teilt er sich dagegen mit David (2 Chron 8:14). Der „Gottesknecht

8 Dazu Matthew J. Lynch, Monotheism and Institutions in the Book of Chronicles. Temple, Priesthood, and Kingship in Post-exilic Perspective, FAT II/64 (Tübingen: Mohr Siebeck, 2013), besonders 72-136. 2 Chron 2:4 als chr Schlüsselstelle „Und das Haus, das ich nun baue, ist gross, denn gross ist unser Gott, mehr als alle Götter“ versteht er im Sinne einer „vertical, or categorical, distinctiveness“ zwischen Gott und Tempel (p. 131), wonach dem Chronisten zufolge „Humans could not add anything to all that Yhwh owned“ (p. 112), so dass der Tempel im eigentlichen Sinne allein auf Gott “as creator” zurückgeht: „The temple was not ... the 'Solomonic' temple”, vielmehr "Yhwh initiated its construction, and provides Solomon with wisdom ... “ (p. 114, vgl. p. 117).

9 Ernst M. Dörrfuss, Mose in den Chronikbüchern. Garant theokratischer Zukunftserwartung, BZAW 219 (Berlin: Walter de Gruyter, 1994), 217. Die Beobachtungen, die Ernst Michael Dörrfuss in seiner so betitelten Untersuchung beibringt, sind sorgfältig zu erwägen, gerade wenn man seine Ansicht, dass Mose ,ausschliesslich in sekundären Texteinheiten” vorkomme und insofern “Mose ... für den Chronisten bedeutungslos”,sei (p. 277), nicht - mehr - teilt.

10 Dörrfuss, Mose in den Chronikbüchern. 
Mose“ beschreibt in den meisten chronistischen Belegen Mose als den, der dem Israel der Wüstengeschichte in JHwHs Auftrag die nötigen Anordnungen für sein Leben vor Gott und in der Welt übermittelt hat. In seiner Person ist also die sonst in Chronik nicht behandelte, aber nichtsdestoweniger normative, Exodus- und Sinaizeit gegenwärtig. Mose ist und bleibt die Instanz, die Israel wie seine kultischen Vollzüge geprägt hat und - wie auch immer - prägen wird.

Genealogisch und im Blick auf die „historische Anciennität“ steht Mose auf einer Ebene mit seinem älteren Bruder Aaron. Durch das ihm anvertraute Wort erscheint er aber als weisungsbefugt, und zwar auch für die opferdienstlichen, das heisst, spezifisch priesterlichen, Belange. Der Gottesdienst, wie David ihn für das neue, auf Dauer erwählte Heiligtum in Jerusalem einrichtet, geschieht „wie geschrieben steht, “ das heisst nach der von Mose vermittelten und schriftlich aufgezeichneten Tradition: Die Priestertora ist in der Mosetora aufgegangen. ${ }^{11}$

Die Institution des Kults ist dadurch charismatisch begründet und eingebettet. Diesbezüglich - und das ist wichtig - zieht die Chronik im Grunde mit ihrer Darstellung der staatlichen Geschichte Israels nur die Linien aus, die bereits in einschlägigen Teilen der - insofern zu Unrecht - als (exklusiv) „priesterlich“ apostrophierten Partien des Pentateuch angelegt sind. Ich beziehe mich hier nachdrücklich auf Levitikus 9-10 nach der eingehenden Analyse von Andreas Ruwe. ${ }^{12}$ Levitikus 9-10, formuliert als „Kopfstück“ jedenfalls die Gesichtspunkte und Leitlinien des anschliessenden Grossabschnitts in Levitikus $11 \mathrm{ff} .^{13}$ Dabei ist Aaron der Inbegriff des Priestertums. Mose dagegen personifiziert die prophetische - oder charismatische - Tradition ${ }^{14}$ mit ihrer Betonung von Auszug, Gerechtigkeit, usw. Die ihm entgegengebrachte Observanz bürgt nach Levitikus 9-10 für die positive Auswirkung des von Israel dargebrachten Kults; Missachtung seiner Anweisungen hingegen führt zu todbringenden Effekten. Was nicht "mosaischer" beziehungsweise - in der Mosenachfolge - „levitischer“ Lehre und Verhaltens-

11 Vgl. zu diesem, "priesterliche” Tora und “prophetisches” Jнwн-Wort einenden, Konzept Mi 4:2b, aufgenommen in Jes 2:3b.

12 Andreas Ruwe, "Das Reden und Verstummen Aarons vor Mose. Lev 9-10 im Buch Leviticus," in Behutsames Lesen, FS C. Hardmeier, ABG 28, ed. Sylke Lubs, Louis Jonker, Andreas Ruwe und Uwe Weise (Leipzig: Evangelische Verlagsanstalt, 2007), 169-96. Es spielt dabei eine untergeordnete Rolle, ob man mit Knohl, The Sanctuary of Silence, bzw. mit Jacob Milgrom in seinem Leviticus-Kommentar (AB 3, 1991) zwischen Teilen einer älteren "Priestly Torah" und einer jüngern "Holiness School” differenziert, obwohl die Ausgangsposition der Chr eher der letzteren entspricht.

13 Ruwe, “Das Reden und Verstummen Aarons vor Mose,” 176.

14 Dazu vgl. Thomas Willi, Die Chronik als Auslegung, FRLANT106 (Göttingen: Vandenhoeck \&Ruprecht 1972), 228-30. 
norm entspricht, ist demgemäss זר. Mit diesem Begriff werden "Elemente im Jerusalemer Kult” bezeichnet, die als „fremdreligiös“ gelten. ${ }^{15}$ Den Textpartien liegt demnach an einem „konstitutionell verfassten Priesteramt,“ das „kultisch auf Mose bezogen“ ist. ${ }^{16}$

Wie eine solche bereits im Pentateuch angelegte „Konstitutionalisierung des Priesteramts“ dereinst aussehen würde, davon vermittelt die in Chronik entworfene Geschichte des davidischen Israel ein lebendiges Bild, vorrangig dank der Rolle, die die Leviten in ihr spielen. Wenn es zutrifft, dass „die P-Texte keineswegs die ungezügelte Macht priesterlicher Interessen“ vertreten und vermitteln, sondern in ihrer Weise den „Impuls des Deuteronomiums“ fortsetzen, ${ }^{17}$ so ist das der Hintergrund und die Motivation für das auffällige und immer beobachtete Interesse der Chr nicht eigentlich an einem besonderen Priestertum, sondern an „den Leviten“ im umfassenden Verständnis.

\section{Levi in Davids neuem Israel}

Denn es ist nicht allein die historisch lange zurückliegende Gestalt des Mose, der mittels seiner Tora für die Kontinuität zwischen dem Gottesdienst am einstigen „Zelt der Übereinkunft“ und dem Gottesdienst am neuen „Haus“ zu Jerusalem bürgt. Sondern dafür steht auch sein Stamm, der Stamm Levi, der durch seinen Umgang mit der Tradition, mit dem Wort und mit der Lehre dem davidischen Jerusalem den Stempel aufdrückt. Was die „Leviten“ in die Waagschale zu werfen hatten, war der geistige Aspekt des an sich rein materiellen Bauunternehmens, wie es der entstehende Tempel darstellt. Gerade dieses einzigartige „Haus“ beruhte auf der Tora als der umfassenden Bildungs-, Rechts-, Religionsund Lebensgrundlage „Israels”. Die Leviten „wachten“ darüber und garantierten durch ihre Existenz, dass schon der Bau wie dann der Kult am Jerusalemer Tempel nicht irgendwie, sondern „gemäss der Tora Moses“ - des Erzleviten! - geschah. ${ }^{18}$

15 Ruwe, “Das Reden und Verstummen Aarons vor Mose,” 181 f. mit Anm. 42.

16 Ruwe, "Das Reden und Verstummen Aarons vor Mose," 186.

17 Ruwe, "Das Reden und Verstummen Aarons vor Mose," 193. Schon Knoppers, "Hierodules, Priests, or Janitors?," 49-72 spricht, speziell im Blick auf Chr, von "a false and misleading dichotomy" (p. 69) zwischen Priestern und Leviten.

18 Zur technischen Bedeutung der Wurzel šmr vgl. die Bemerkung bei David Talshir, "A Reinvestigation of the Linguistic Relationship between Chronicles and Ezra-Nehemiah," VT 38 (1988): 177: Im Unterschied zu ma hị ${ }^{a}$ qet ist "mišmeret a word connoting the fulfilment of a certain cultic role or the appointement to such a role". 
Der Übergang vom mobilen zum stabilen Heiligtum betrifft - immer laut Chr - ganz speziell den Stamm Levi. In ihrer Rolle verkörpern die Leviten sozusagen die Kontinuität in der Diskontinuität. Das spiegelt sich in verschiedenen kleineren wie grösseren Eigentümlichkeiten im Aufriss von 1. Chronik. So eröffnen beispielsweise die Leviten - nicht etwa die (natürlich in den Stamm Levi integrierten) Kohanim, die Priester - das Bevölkerungstableau des gegenwärtigen und damit auch künftigen Israel, das David zu Ende seiner Lebzeiten Revue passieren lässt (1 Chron 23). Ihre prominente Rolle ist aber mit 1. Chronik 5:27-6:66 bereits in einer Bestandesaufnahme aus früheren Zeiten, in den „Bürgerrechtslisten” Israels (1 Chron 1-8, 9), präludiert. Schon hier nimmt Levi - nicht Aaron, nicht die Priesterschaft - eine zentrale Stellung ein: Levi ist die Mitte, das Herz des chronistischen Ganz-Israel. Mit seiner Einordnung und Anordnung Levis geht der Chronist dort schon seinen eigenen und neuen Weg. Seine Konzeption unterscheidet sich dabei von den älteren Darstellungen Levis, obwohl diese zum Teil auch die Quellen für 1. Chronik 6 sind. ${ }^{19}$ Wo nach Numeri 1:49-53; 26:57-62 und Josua 13-19 Levi gerade nicht „inmitten“ (בתוך) Israels, sondern als Grösse sui generis berücksichtigt werden soll, ${ }^{20}$ da gehört Levi nach dem chronistischen Konzept im Gegenteil mitten ins zwölfstämmige Israel hinein. Levi wird nicht im Zusammenhang mit Jerusalem, nicht wie in der Priesterschrift zusammen mit dem Heiligtum und dem Kult behandelt, sondern als vollgültiger und gleichberechtigter Teil des Volkes! Levi ist in der Königszeit von David an, der Chronik zufolge, etwas anderes geworden als in der Priesterschrift. Aus dem ausgesonderten Stand von Kultsachverständigen der Frühzeit ist ein ins zwölfteilige Israel eingegliederter Stamm geworden. ${ }^{21}$ Für den Chronisten ist Levi eine, ja die Funktion Israels, Levi verkörpert Israel at its best, und umgekehrt ist Israel das Volk Levis. ${ }^{22}$

19 Am ehesten liesse sich 1 Chron 6 noch mit Ex 6:14-27 vergleichen, wo die levitischen Sippen an die Familienhäupter Rubens und Simeons angeschlossen werden, allerdings nicht als deren Mittelpunkt, sondern mit Achtergewicht auf Mose und Aaron.

20 Num 1:49-53 trennt Levi ausdrücklich vom übrigen Israel; nicht anders Num 3 als Nachtrag zur Ordnung der Israelstämme von Num 2. Num 26:57-62 ist ein Sonderanhang zu den bereits komplett aufgezählten Sippen der Israelstämme, und in Jos 13-19 fehlt Levi überhaupt und wird deshalb in Jos 21 separat abgehandelt. Ganz anders 1 Chron 6 und schliesslich erst recht 1 Chron 23:6-24:31 im Rahmen des in 1 Chron 23-27 geschilderten davidischen Israel - kein Mensch käme hier auf den Gedanken, mit Num 1:49 oder 2:33 zu behaupten, dass Levi nicht gleich allen anderen Angehörigen Israels “mitten unter den (übrigen) Israeliten zu berücksichtigen” sei.

21 Dazu ausführlicher Willi, BK XXIV/1, 197-99.

22 Dass dadurch eine auf die geschichtliche Ebene herabgebrochene Verbindung zu dem Konzept des “priesterlichen” Textes Num 3:11-13, nach dem die Leviten die Stelle der "ausgelösten" erstgeborenen Israeliten einnehmen, ist nicht zu verkennen. 
Die hier berührten Vergleichs- beziehungsweise Quelltexte entstammen dem Pentateuch. Leviten begegnen zwar auch in Esra-Nehemia. Aber nur der Pentateuch und Chronik bemühen sich, je auf eigene Weise, die zweifellos lange zurückreichende Geschichte des Levitums und des Levitismus systematisch einzuordnen, und einzig Chronik gewährt den alten Überlieferungen schon in den Bürgerrechtslisten Raum und sucht sie nun für die Darstellung der staatlichen Geschichte Israels fruchtbar zu machen. Gerade darin erweist sich der Chronist als umsichtiger Historiograph, der aus den alten Nachrichten als Quellen schöpft und die einschlägigen Texte neu $\mathrm{zu}$ lesen und $\mathrm{zu}$ exegesieren weiss, um sein Tableau über David und die Davidszeit zu entwerfen.

Die chronistische Auffassung von Levi hängt dabei mit dem alten, vorexilischen Stand Levi nur sehr indirekt zusammen. ${ }^{23}$ Und auch mit der realen Situation und Funktion von Leviten im Betrieb des zweiten Tempels ist sie kaum deckungsgleich. Was einst zu den hervorragendsten charismatischen Phänomenen nordisraelitischen religiösen Lebens gehört hatte, war durch die Entwicklung der Umstände dreifach bis ins Mark ihrer Existenz getroffen. Israel, das Nordreich, hatte als selbständiges Gemeinwesen aufgehört zu existieren. Im Süden liefen die Reformmassnahmen Josias auf eine Stärkung der Priesterschaft Jerusalems hinaus. Zu ihr gehörte dann auch der von Haggaj und Sacharja erwähnte Seniorpriester Josua ben Jozadak, mit dem sich der Wiederaufbau des Heiligtums verbindet. Leviten erscheinen hier und auf lange hinaus überhaupt nicht auf der Bildfläche. ${ }^{24}$ Das Levitentum war keine condicio sine qua non für die Ausübung

23 Zum Folgenden vgl. einführend und begleitend Thomas Willi, "Leviten, Priester und Kult in vorhellenistischer Zeit. Die chronistische Optik in ihrem geschichtlichen Kontext," in Gemeinde ohne Tempel/Community without Temple. Zur Substituierung und Transformation des Jerusalemer Tempels und seines Kults im Alten Testament, antiken Judentum und frühen Christentum, WUNT 118, ed. Beate Ego, Armin Lange und Peter Pilhofer, (Tübingen: Mohr Siebeck, 1999), 75-98, vor allem 89-95. Für die Leviten der Vor- und Frühzeit existieren nur ganz wenige aussagekräftige Nachrichten, und man wird Jaeyoung Jeon, "Levites: I. Hebrew Bible/Old Testament," in EBR 16 (2018) Sp. 336-46 nur zustimmen können, wenn er resümiert, dass "no consensus has yet been made among critics concerning the date and origin of the Levites or Levitical priesthood" (Sp. 339).

24 Signifikant für die Zurücksetzung der Angehörigen levitischer Gruppen und Familien ist die Evidenz aus dem 6. Jahrhundert in Ez 44 und aus der Mitte des 5. Jahrhunderts in Esr 8. Esra ist sich nach diesem Kapitel der Bedeutung des levitischen Elements für das neue Jerusalem insofern bewusst, als er die Karawane nicht aufbrechen lässt, bis er ein wenn auch höchst bescheidenes Kontingent von rückkehrwilligen Leviten beisammen hat. Die Zeiten waren längst vergangen, wo jemand wie in Ri 18:3f. qua Levit und dank seiner Vertrautheit mit Überlieferung und Gesang zum Priesterdienst qualifiziert war. Gewiss mochte es einzelne Priester geben, die sich der levitischen Tradition verpflichtet wussten. 
der priesterlichen Funktionen. Der Jerusalemer Klerus war eine lokale Priesterschaft unter Hunderten, ja Tausenden anderer im weiten achämenidischen Reich. Sollte er „israelitisch“ im Sinne der Konformität mit Tradition und Schrift sein, dann konnte und durfte er aber auf das levitische Element nicht verzichten.

Das Zeugnis des Maleachibuches verrät manches von dieser Spannung zwischen Realität und Norm, zwischen gegenwärtiger Wirklichkeit und altehrwürdigem Ideal, zwischen Praxis und - vielleicht auch hier schon weitgehend schriftlicher - Tradition. Eine sorgfältige syntaktische Untersuchung kommt zu dem Schluss: „While the Book of Malachi equates the function of kohen and lewi bene-lewî the book offers no contextual ground by which to explain this equation. “ ${ }^{25}$ Ein Stamm wie die anderen Stämme zu sein, setzte familiäre, ortsbürgerliche, landschaftliche, territoriale Bindungen voraus. Und genau diese fehlten dem charismatischen und oft auch nomadisierenden Levitentum der Frühzeit - sein „Anteil“ war JHwH, wie es die alte Levitenregel Exodus 32:29 im Positiven wie im Negativen festhält: „Da sprach Mose: Füllt heute eure Hände zum Dienst für den HERRn - denn ein jeder ist wider seinen Sohn und Bruder gewesen - damit euch heute Segen gegeben werde.“ (Vgl. auch den Levi-Segen in Dtn 33:8).

Wenn also, wohl im ausgehenden 5. Jahrhundert v. Chr., die chronistische Darstellung der Geschichte Israels ab David die Leviten als eine Art „Stamm der Stämme“ Israels behandelt, so hängt das mit der Eigenart der chronistischen Historiographie zusammen. Nicht das DtrG ist für die Chronik bei ihrem Geschichtsentwurf massgebend, sondern - und das ist etwas durchaus Anderes - die in ihm aufbewahrten und von ihm tradierten Überlieferungen und Materialien. Aus chronistischer Optik handelt es sich bei dem, was heute als DtrG bezeichnet wird, um ein Florilegium diverser Quellen und Nachrichten aus der Feder von Propheten unterschiedlicher Epochen und Regionen.

Diese auch literarisch fassbare Hochschätzung ${ }^{26}$ der Boten Gottes macht es von vorneherein unwahrscheinlich, dass entsprechende Überlieferungen und Äusserungen aus einer der behandelten Zeit vorangehenden Epoche einfach übergangen, ja eskamotiert und gezielt verdrängt sein sollten. Auch wenn sie nicht als prophetisch anzusprechen sind, so haben sie eigentlich nicht weniger, sondern im Grunde noch mehr Anspruch auf Autorität und Gültigkeit. Was Mose in seiner ihm aufgetragenen Tätigkeit und Tora, was Aaron in seiner Einrichtung

25 Julia M. O’Brien, Priest and Levite in Malachi, SBLDS 121 (Williston, VT: SBL Press 1990), 47, wo auch verschiedene Versuche zur Lösung des Problems genannt sind.

26 Vgl. Thomas Willi, Die Chronik als Auslegung, FRLANT 106 (Göttingen: Vandenhoeck \& Ruprecht, 1972), 216-41. 
des Kults einst gebracht haben und für Israel auf Dauer bedeuten, ist und bleibt nicht nur unvergleichlich, sondern schlicht elementar.

Genau diese bleibende Gültigkeit bringt es mit sich, dass die - nach moderner Nomenklatur - pentateuchische Überlieferung mit ihren so vielfältigen Facetten (den sogenannten vor- wie nachexilischen „Quellenschriften“) eine besondere Qualität aufweist, last not least für die Chronik, die sich zwar explizit überhaupt nicht mit diesen Zeiten und Dingen befasst, implizit dagegen um so mehr auf sie bezogen bleibt. ${ }^{27}$ Gerade weil der Pentateuch die Grundlagen der Entstehung und Entwicklung des Israels der zwölf Stämme dokumentiert, sieht sich der Chronist als Historiker vor die Frage gestellt, wie diese Grundlagen bewahrt und im Laufe der Geschichte von Fall zu Fall neu realisiert werden, wenn sich die Voraussetzungen ändern. ${ }^{28}$ Der Chronist musste mit dem Kern des Pentateuchs in seiner Letztfassung davon ausgehen, dass die „Stiftshütte“ das „Zelt der Übereinkunft/Begegnung, “ die Mitte nicht bloss des kultischen Geschehens, sondern des Lebens der Israelstämme insgesamt bildete. Dann erhob sich folgerichtig die Frage: Inwiefern ist der Jerusalemer Tempel noch das alte Heiligtum, und inwiefern ist er es nicht? Was ist aus dem reich ausgebauten Gottesdienst in der Wüste und mit denen, die ihn verantworteten und ausführten, eigentlich geworden? ${ }^{29}$

Genau diese in Israels Geschichte eingetretene Transformation hat der Chronist im Blick. In einprägsamer Bildhaftigkeit macht er sie an einer ganz bestimmten Stelle, anhand des Wandels in der Funktion der Leviten dingfest. Früher hatten sie nach Deuteronomium 10:8 rein physisch die Aufgabe, die Lade zu tragen, wie die Erzählung von der Überführung der Lade in 1. Chronik 15:2, 15, 26 f. einschärft. Nun aber, da diese ihren festen Platz gefunden hat, sind die Leviten mit der Überlieferung und dem Wort betraut, sollen die Tora anwenden und die Schrift aus-

27 Auch die pentateuchischen Überlieferungen sind für den Chronisten nicht als - gar im Skopus unterschiedene - Konzepte im Sinne von "Quellenschriften" von Belang, sondern als Erzählungen und Anweisungen aus Israels Frühzeit von Belang. Was etwa seine Haltung gegenüber "dtr" versus "priesterlicher" Traditionen angeht, so wird man mit Knoppers, "Hierodules, Priests, or Janitors?,” 68 sagen müssen: "The Chronicler is heir to and interpreter of both traditions."

28 Daher ist der der Chronik gemachte Vorwurf, sie beschreibe ihr Israel nach dem Bild des Pentateuchs, vorab seiner insgesamt "priesterschriftlich" gestalteten Endfassung, weitgehend unbegründet, und man wird Julius Wellhausen nicht einschränkungslos folgen, wenn er behauptet, dass im DtrG die Vergangenheit verurteilt werde, während dagegen "in der Chronik ... die Geschichte des alten Israel nach Massgabe des Priesterkodex umgedichtet” werde. Sie disqualifiziere sich in ihrer Geschichtsdarstellung als williges Vollzugsorgan kultisch motivierter Programmatik. Dem Chronisten als Historiker geht es wesentlich darum, alle ihm vorliegenden Überlieferungen, Nachrichten, Quellen zu berücksichtigen. Er kennt und anerkennt sodann - und auch das gehört zum Rüstzeug der Geschichtsschreibung - die Differenz der Zeiten und Epochen. 29 Explizit stellt sich der Chronist dieser Frage in 2 Chron 1:3f. 
legen. In bemerkenswert gegensätzlich-paralleler Formulierung zu 1. Chronik 15:2 hält aber nun David am Ende seines Lebens als Vermächtnis fest:

\begin{abstract}
${ }^{25}$ Denn David sagte: JHwH, der Gott Israels, hat seinem Volk Ruhe verschafft und in Jerusalem Wohnung genommen auf Weltzeit. ${ }^{26}$ So haben auch die Leviten nicht mehr das Wohngemach und all seine Geräte zu seinem Dienst $z u$ tragen. ${ }^{27}$ Aber tatsächlich sind sie in den letzten anordnenden Worten Davids (da, und zwar) als Zahl der Leviten im Alter von zwanzig Jahren und darüber, ${ }^{28}$ denn ihre Stellung ist an der Seite der Söhne Aarons, zum Dienst am Hause JHwHs ...
\end{abstract}

Diese „Wahrung“ der Tradition, von der 1. Chronik 23 abschliessend mit Verwendung der hebräischen Wurzel שמר spricht (V. 32), stellt die Fortführung der alten Levitenobliegenheiten unter den neuen Bedingungen des festen Heiligtums dar und weitet die physischen Verrichtungen aus, indem sie vergeistigt als Handhabung des Wortes, der Tora, ja der Schrift, erscheinen. ${ }^{30}$ Aus dem Stand der charismatisch inspirierten Outsiders ist in Chronik vermittels kreativer Auslegung ${ }^{31}$ der Stamm Levi geworden, dem der grundsätzlich gleiche Auftrag obliegt, jetzt freilich als Herz des Zwölfstämmevolks.

Die Leviten, an ihrer Spitze Mose, „wachten“ darüber, dass der Kult nicht einfach als opus operatum vollzogen wird, sondern dass er nach der Israel anvertrauten Art, der Mose von Gott zuhanden Israels übergebenen und als Schrift vorliegenden Grundlage geschieht! Darauf bezieht sich die Schriftkonformität des jeweiligen Sachverhalts. Diese Schriftkonformitätsklauseln (nicht Zitationsformeln!) betonen die Selbigkeit der Autorisierung, die Identitätsstiftung zwischen der grundlegenden Offenbarung von einst und ihrer Realisierung von jetzt, wie sie in Chronik auf David zurückgeführt wird. Im Rückgang auf Mose betont Chronik (wie auch Esr-Neh), diese oder jene Massnahme sei nicht zufällig oder arbiträr nach menschlichem Gutdünken vollzogen worden, sondern sei geschehen kakkatuv, „wie geschrieben - nämlich nieder- geschrieben und vor- „geschrieben“steht“. ${ }^{32}$

30 Vgl. den Versuch von M. Gertner, "Masorah and Levites. An Essay in the History of a Concept,” VT 10 (1960): 242-72, das frühjüdische Levitenamt über einen Rückschluss von den mittelalterlichen Masoreten auf die persisch-hellenistischen Verhältnisse zu bestimmen und in den „medieval masoretic masters“ geradezu ein „revival ... of the earlier, pre-talmudic, ideals“ $\mathrm{zu}$ erkennen (p. 242).

31 Nicht anders werden auch die Texte von Qumran ihr Bild von den Leviten mittels einer „simply text-centered creative exegesis“ entwerfen, vgl. Harald Samuel, „Levites: II. Judaism, “ in EBR 16 (2018): Sp. 348.

321 Chron 16:40; 28:19; 2 Chron 23:18; 30:5, 18; 31:3; 35:4, 12, 26; in etwas anderer Weise 1 Chron 24:6. Die Ausdrucksweise wird variiert a) in der Formulierung „nach (dem Buch, der Verschrif- 
Die Verschriftung der dem Mose mündlich erteilten Tora ist sozusagen die technische Seite der Sache. Davids Vorbereitungen für Heiligtum und Kult hängen aber mit der Grundgeschichte der Wüstenzeit nicht nur dadurch zusammen, dass sie über die schriftliche Fassung mit dem göttlichen Wort zusammenstimmen, sondern auch dadurch, dass nun in der Linie des Mose auch sein Stamm für dessen Geltung bürgt. Die Personalisierung durch Levi begleitet und schützt sozusagen die Skripturalisierung der Tradition.

\section{5 "Grundgeschichte" und Chronik nach chronistischem Selbstverständnis}

Von diesen Voraussetzungen her gestaltet der Chronist seine Geschichtserzählung. Weil er die Zeit des Tempels als eine von der Väter- und Exoduszeit grundsätzlich unterschiedene kennt und beschreibt, sieht er sich vor die Aufgabe gestellt, dem fundamentalen Übergang, der sich mit David vollzieht, und dem Altüberlieferten, das da neu wird, nachzuspüren. Das historische Bewusstsein für den Bruch der Zeiten - der im DtrG so nicht existiert - macht es möglich und nötig, eine neue Darstellung der Geschichte zu schreiben.

Die alten Überlieferungen zur Schöpfung und den Vätern, zum Exodus und zur Wüstenzeit in ihrer ganzen Vielschichtigkeit haben nicht erst die moderne Forschung angezogen und irritiert. ${ }^{33}$

tung) der Weisung JHwhs durch Mose“: 2 Chron 23:18; 2 Chron 25:4 (zit. Dtn 24:16); 30:16; ähnlich 33:8; dann 34:14 und 35:12; b) mit der Betonung „ganz wie Mose [...] geboten hat“: 1 Chron 6:34; 15:15; etwas modifiziert 22:13 und 2 Chron 25:4; c) schliesslich auf der Ebene der Ausführung (nicht der Übermittlung und daher ohne Bezug auf Mose) „,nach dem Gebot/der Regelung/der Vorschrift Davids (und Salomos)”, aber auch “des Königs (sc. Hiskia oder Josia)“: „Nach dem Gebot (mizwa)“: Neh 11:23; 12:24, 45; 2 Chron 8:13, 14 (mit mischpat), 15; 29:15 (mit dibre JHWH), 25bis; 30:12 (mit debar JHwH); 35:10, 15, 16. Bzw. Verbalwurzel zwh: Esr 4:3; Neh 8:1, 14; 1 Chron 6:34; 15:15; 16:40; 22:6; 24:19 (neben mischpat; 2 Chron 25:4 // 2Kön 14:6. „Nach der Regelung (mischpat)“: Esr 3:4; Neh 8:18; 1 Chron 6:17; 15:13 (mit darasch); 23:31; 24:19 (neben ziwwah); 2 Chron 4:7, 20; 8:14 (neben mizwa); 30:16 (neben tora); 35:13. „Nach der (Vor-)Schrift (ketab)“: 1 Chron 28:19; 2 Chron 2:10; 35:4. Das aramaisierende Substantiv ketab begegnet weitaus am häufigsten im Buch Esth.

33 Das zeigt sich schon am Spektrum ihrer Bezeichnungen: Rein technisch ist von Bereschit, Schemot usw., elementar ist von hakkatuv, dem „schriftlich Festgehaltenen“ oder ebenso allgemein von sefär, ,Verschriftung“ die Rede, die nach dem Übermittler als ספר משה, nach der Gattung als ספר תורה, präzisiert wird, die sich später zum Pentateuch als dem Fünf-Buch zusammenfassen lassen. 
Auch der Chronik sind diese Überlieferungen, paradoxerweise gerade weil sie nicht ihr Thema sind, alles andere als eine bedeutungslose Nebensache. Im Gegenteil: Dank der chronistischen Historiographie und ihrer Anlage gewinnen sie eine ganz neue Qualität. Sie bilden die unabdingbare Voraussetzung, die Basis und den Hintergrund für die mit David anhebende Geschichte Israels im Zeitalter des Ersten Tempels, gerade dort, wo es um ihre Transformation geht. Für die Geschichte, die nach Chronik mit David neu beginnt, sind jene nicht thematisierten, wohl aber selbstverständlich vorausgesetzten Traditionskomplexe sozusagen die Grundgeschichte im Sinne einer basic story. ${ }^{34}$

Eine solche Grundgeschichte ist etwas anderes als eine Gründungs- oder eine blosse Vorgeschichte, die die Voraussetzungen für das, was danach kommt, klärt, selber aber abgetan und vergangen und insofern bedeutungslos ist. Die Grundgeschichte, das, was in ihr ,aufgeschrieben“ ist, bildet vielmehr die Referenz, den Massstab für die neue, anders gelagerte Zeit: jene des - in diesem Falle - Ersten Tempels, des davidisch-salomonischen Jerusalemer Heiligtums, in dem das einstige mobile Ladeheiligtum sein Ziel und Ende gefunden hat und den Anspruch erhebt, als Residenz des Weltreichskönigs zu dienen.

34 Der Ausdruck Grundgeschichte ist als solcher nicht neu. Wenn er auch in der neueren alttestamentlichen Wissenschaft, vorab in ihrer Form- und Literaturgeschichte, u.W. bislang nicht angewendet wurde, so hat er doch seinen Platz im werdenden historischen Denken der Barockzeit und vor allem dann in der beginnenden universalgeschichtlichen Betrachtungsweise des 19. Jahrhunderts, anders ausgedrückt im Umfeld der Untersuchungen Wilhelm Martin Leberecht de Wettes oder Karl Heinrich Grafs und im Vorfeld der darauf aufbauenden Entwürfe Julius Wellhausens. So spricht etwa der christliche Kabbalist Christian Knorr von Rosenroth (1631-1689) im Blick auf Ri 11: „Die Grundgeschichte von des Jephtha Tochter lautet ...“ (Des vortrefflichen Engelländers Thomae Brown ... Psevdodoxia Epidemica. Das ist Untersuchung derer Irrthümer, so bey dem gemeinen Mann ... im Schwange gehen: In Sieben Büchern ... und dann ferner in denen übrigen sechs Büchern von den Irrthümern, die Mineralien, Gewächse, Thiere ... betreffend. Aus dem Englischen und Lateinischen übersetzt durch Christian Peganium, in Teutsch Rautner genannt [Frankfurt a.M. 1680]: p. 820). Bei Ernst Moritz Arndt bezeichnet der Begriff „das Unmittelbare und Ursprüngliche, was als Grundgeschichte des Lebens der Völker in der Fabel und dem Mythos liegt“ (Schriften für und an seine lieben Deutschen, Band 4 [Berlin: Weidmann, 1855]: p. 305). Nach Friedrich Schlegel in seiner Philosophie der Geschichte haben „die tieferen Forschungen ... wiederholt hingewiesen” auf "diese Grundgeschichte ... der Griechen.“ (Philosophie der Geschichte 1: Sämtliche Werke Bd. 13 [Wien: Mayer 1846]: p. 223). 